\title{
Attractive Colloidal Rods in Shear Flow
}

\author{
M. Ripoll, * P. Holmqvist, R. G. Winkler, G. Gompper, J. K. G. Dhont, and M. P. Lettinga \\ Institut für Festkörperforschung, Forschungszentrum Jülich, D-52425 Jülich, Germany
}

(Received 19 March 2008; published 15 October 2008)

\begin{abstract}
The effect of shear flow on the isotropic-nematic phase transition of attractive colloidal rods is investigated by a combination of simulations and experiments. The isotropic phase aligns with the flow, while the nematic phase undergoes a collective rotational motion which frustrates the merging of the coexisting regions. The location of binodals, spinodals, and the tumbling-to-aligning transition line in the shear-rate versus concentration plane are investigated. The phase diagrams in the shear-concentration plane for the various strengths of attractions can be mapped onto a master curve by appropriate scaling.
\end{abstract}

DOI: 10.1103/PhysRevLett.101.168302

PACS numbers: 82.70.- $-\mathrm{y}, 64.60 .-\mathrm{i}, 83.50 .-\mathrm{v}$

Phase transitions occurring in soft matter systems are significantly affected by flow. Both the nature and location of the phase transition lines are changed due to the applied flow [1]. The challenge is to find the parameters that determine the nonequilibrium steady states under flow conditions. One important parameter is the interfacial tension between coexisting phases [2], as is the case for crystal and liquid phases of spherical colloids under shear flow [3]. Colloidal-rod suspensions constitute a particularly interesting system to study the effect of flow on their phase behavior [4,5], since rod orientation is strongly coupled to the shear field. Rods in the isotropic $(I)$ phase, align with the flow and become paranematic $(P)$. This suggests that the transition to the nematic $(N)$ phase, where rods have orientational order, is facilitated by shear. On the other hand, rods in the nematic phase undergo a collective tumbling motion in the presence of shear flow [6-8]. The question that then arises is how these two effects will affect isotropic-nematic coexistence. Apart from fundamental interest, a detailed understanding of the flow behavior of a model system of attractive colloidal rods is useful for industrial applications where shear alignment of elongated objects, such as carbon nanotubes [9], wormlike micelles [10], and polymers, play a role.

In this Letter, the nonequilibrium phase diagrams of attractive colloidal rods in shear flow are investigated by a combination of mesoscale hydrodynamic simulations, small-angle light-scattering (SALS) experiments, and rheology. The simulations allow for a microscopic understanding of the behavior of coexisting phases and their interface under shear, including the possible role of collective tumbling motion of rods. In addition, the binodals-which define the concentrations of coexisting paranematic and nematic states in the shear-rate versus concentration plane-are determined. Experiments allow for the determination of the full phase diagrams, including spinodals-where the homogeneous nematic state becomes unstable-binodals, and tumbling-to-aligning transition lines. Both in simulations and experiments, the attractive rod-rod interactions are systematically varied, which affects the phase behavior, interfacial properties of coexisting phases as well as tumbling behavior.

Earlier experimental studies have mostly focused on the shear-induced $P-N$ transition of wormlike micelles [10], that can break and recombine. For dispersions of hydroxypropyl-cellulose, the $P-N$ transition temperature has been shown to change due to shear flow [11]. Much less is known about suspensions of monodisperse rodlike colloids like $f d$-virus particles. These systems exhibit an $I-N$ phase transition without flow. The $I-N$ biphasic gap width in the absence of flow is known to increase significantly when polymer is added, which induces depletion attractions [12]. The nonequilibrium binodal under shear flow conditions for a single, fixed strength of attraction has been studied by rheological experiments on $f d$-virus dispersions, which show that the $P-N$ transition concentration changes on applying flow [13]. Hence, both attractive interactions as well as flow have a pronounced effect on the location of $P-N$ transition lines. Also the effect of shear on nematicparanematic interfaces in systems of soft repulsive ellipsoids has been studied by simulations [14].

In our simulations, each rod consists of $N_{m}$ monomers with a bond and a bending potential. The bond potential keeps the distance between monomers essentially fixed at $l_{b}$ while the bending potential provides rigidity to each rod [15]. The interactions between the monomers of different rods are described by a Lennard-Jones potential with a minimum of $\epsilon$ (in units of the thermal energy $k_{B} T$ ) and a diameter $\sigma$. Molecular dynamics simulations of rodlike colloids are combined with a mesoscopic description of the solvent known as multiparticle-collision dynamics (MPC) (see Ref. [16] for details). The simulation box consists of $20 \times 22 \times 30$ cubic collision cells with lattice constant $a$, and 50 solvent particles per cell. We use the parameters $\sigma=l_{b}=a / 4$. This hybrid approach has been shown to account for long-range hydrodynamic interactions between rods [15] and has been successfully applied to different systems in flow [17]. Lubrication forces, however, are not accounted for in MPC simulations. The rods have an aspect ratio of 20. Previous simulations indicate 
that $I-N$ coexistence for such rods occurs in a small interval of packing fractions $\phi_{0}$ around 0.125 [18], which we obtain by including $10^{4}$ rods in the simulation box. To impose shear, Lees-Edwards boundary conditions are employed. For a homogeneous fluid, these boundary conditions result in a linear velocity profile $\left(\boldsymbol{v}_{x}, \boldsymbol{v}_{y}, \boldsymbol{v}_{z}\right)=$ $(\dot{\gamma} y, 0,0)$, with $\dot{\gamma}$ the applied shear rate (where the $x$ direction is the flow direction and the $y$ direction is the gradient direction). Shear rates are expressed in simulation time units $\sqrt{m a^{2} / k_{B} T}$, where $m$ is the mass of a solvent molecule.

The simulated system is first prepared in equilibrium with coexisting isotropic and nematic phases, where the director of the nematic phase is aligned parallel to the interface. A snapshot of $I-N$ coexistence in equilibrium is shown in Fig. 1(a). Then, shear is applied with the imposed flow direction parallel to the interface. At small shear rates, rods in the isotropic phase flow-align, i.e., become paranematic, and portions of the nematic phase display collective rotations, as shown in Fig. 1(b) and in Ref. [19]. These periodic motions were also observed in simulations for hard rods in a full nematic phase [20]. This behavior is characterized by both the local concentration
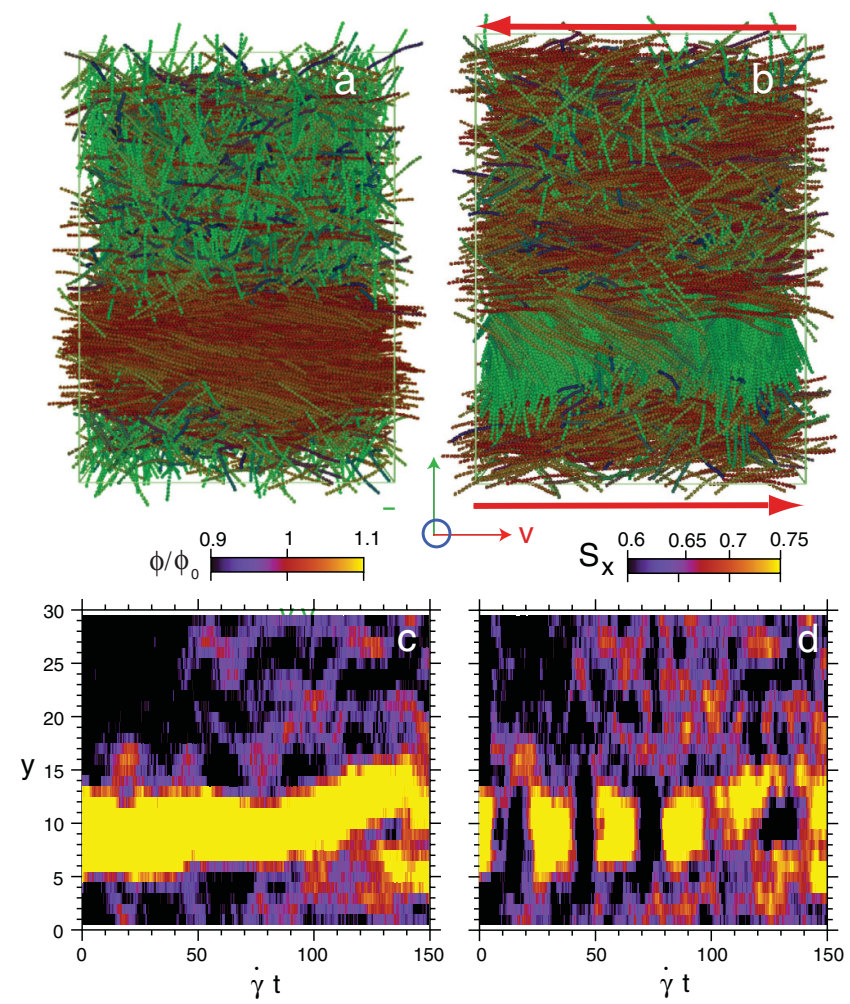

FIG. 1 (color). Snapshots of the simulation box with $\epsilon=3.5$, (a) at equilibrium, and (b) in a tumbling event at $\dot{\gamma}=0.003$. Colors in (a) and (b) are coding the rod orientation: horizontal is red, vertical is green, and perpendicular to plane of view is blue. Red arrows in (b) denote flow direction. (c) Time evolution of the normalized density $\phi / \phi_{0}$, and (d) of the orientational order parameter $S_{x}$ along the gradient direction. For a movie see Ref. [19]. and the local orientational order parameter $S_{x}(y) \equiv$ $\left[3 \hat{u}_{x} \hat{u}_{x}-1\right] / 2$, where $\hat{u}_{x}$ is the component of the unit vector connecting the end points of a rod along the flow direction, and the overline indicates averaging over the vorticity and flow directions. The time dependence of the density $\phi$ and orientational order parameter $S_{x}$ of rods as a function of the position $y$ along the gradient direction is plotted in Figs. 1(c) and 1(d). As can be seen from Fig. 1(c), the nematic phase has a higher concentration than the isotropic phase, as expected. More importantly, Fig. 1(d) demonstrates the periodic tumbling motion of rods in the nematic phase. This is seen for all nematic domains in coexistence with paranematic regions and for all strengths of attractions studied. At long times, both Figs. 1(c) and 1(d) show that the nematic phase is somewhat displaced and seems to split into two nematic domains. Such a behavior is also seen experimentally by confocal microscopy [19]. Binodals are determined at times where the density of the paranematic state has reached a stationary value (this happens approximately at $\dot{\gamma} t=25,50,100$ for $\epsilon=3.0,3.2,3.5$, respectively) by averaging over about one tumbling period. The binodals obtained in this way are plotted in Fig. 2(a) for different attractions. Here, the concentration is expressed in terms of $\varphi_{\text {nem }}$ and the shear rate is scaled by a factor $\dot{\gamma}_{\max }$ such that all data points fall onto a master curve. This master curve is obtained from the combined set of data from both simulations and experiments (which will be discussed below). The scale factor $\dot{\gamma}_{\max }$ can be identified with the maximum of the binodal. Simulations for shear rates just above $\dot{\gamma}_{\max }$ indeed indicate a homogeneous state. The inset in Fig. 2(a) shows that the effect of the increasing attraction between rods is that the coexistence region widens and that $\dot{\gamma}_{\max }$ increases. Because of slowing down of the dynamics and large-scale correlations close to spinodals, considerably longer simulations with larger system sizes would be required to determine the location of spinodals.

In our experiments, we use $f d$-virus suspensions where depletion attractions are varied through addition of dextran. $f d$ virus is a long and thin rodlike particle $(880 \mathrm{~nm}$ long, aspect ratio 120, persistence length $2.2 \mu \mathrm{m}$ ) [12]. Suspensions of varying colloid and dextran concentrations were prepared as follows. First, a homogeneous $f d$-virus suspension of $21.1 \mathrm{mg} / \mathrm{ml} f d$ virus with dextran $(480 \mathrm{kd}$, Pharmacosmos) in $20 \mathrm{mM}$ Tris buffer with $100 \mathrm{mM} \mathrm{NaCl}$ is allowed to macroscopically phase separate into an isotropic and nematic phase. A volume $V_{\text {nem }}$ of the nematic phase is then mixed with a volume $V_{\text {iso }}$ of the coexisting isotropic phase. The concentration of the $f d$ rods is characterized by the fraction $\varphi_{\text {nem }}=V_{\text {nem }} /\left(V_{\text {nem }}+V_{\text {iso }}\right)$ of the nematic phase that is present in the homogeneous mixture. This ensures that the polymer osmotic pressure (and hence the strength of attraction) in the homogeneous suspensions is independent of the rod concentration like for the simulations. The way the phase diagram is determined experimentally is conceptually different from simulations. We 


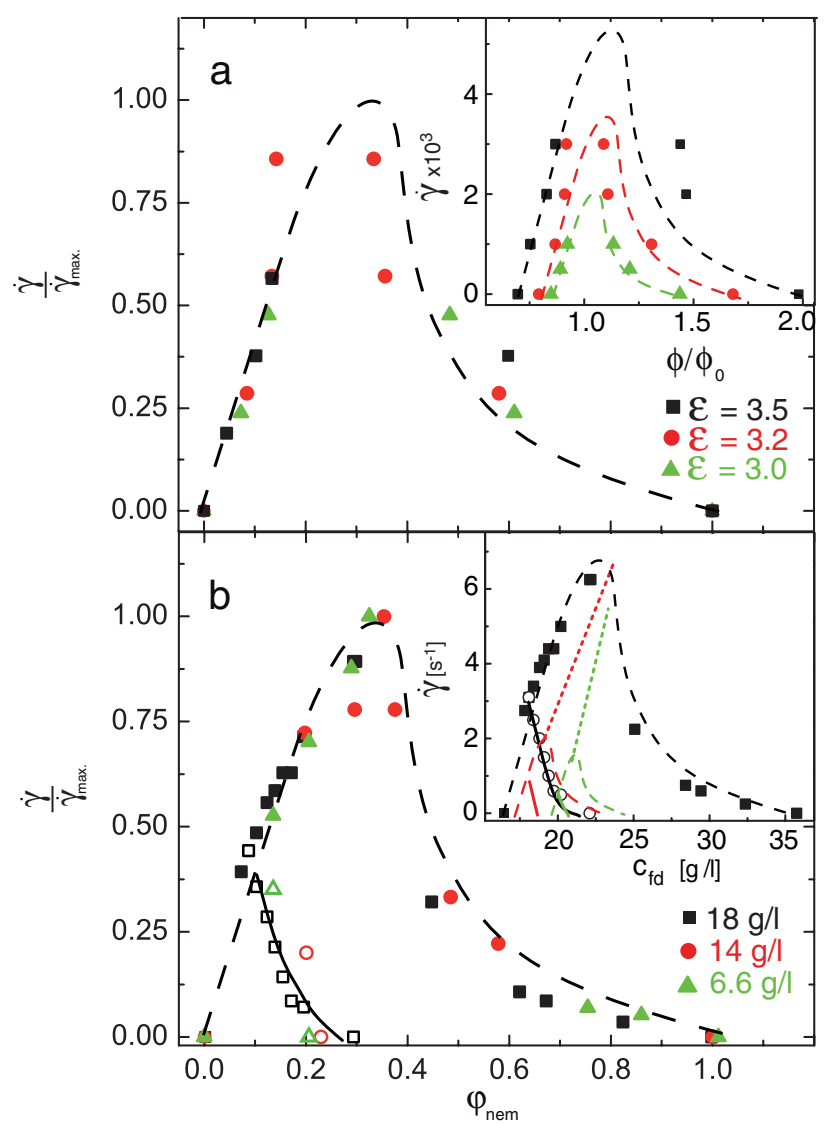

FIG. 2 (color online). (a) Nonequilibrium phase diagram obtained from simulations and (b) from experiments for various values of the strength of attraction interaction, with shear rates normalized by the maximum shear versus the fraction of equilibrium nematic phase. The open symbols in (b) are spinodal points. The insets present the unscaled data. Data points for the lower binodals in the inset in (b) are not shown for clarity. The dotted lines originating from the maximum of the binodal in the experimental diagram correspond to the tumbling-to-aligning transition lines. The solid lines in the inset in (b) at low shear rates indicate the location of the spinodals for the two lowest polymer concentrations. The dashed lines are the conjectured master curve, which are identical in (a) and (b).

obtain the binodal and spinodal experimentally by starting from a stable, homogeneous shear-induced nematic state at $100 \mathrm{~s}^{-1}$, and taking data immediately after reducing the shear rate to a prescribed value. We measure the induction time for the formation of inhomogeneities, as probed with light scattering, after such a shear-rate quench. The induction time vanishes on approaching the spinodal line, while it diverges on approaching the binodal. In Figs. 3(a) and 3(b), the induction time and its inverse are shown as a function of concentration for several shear rates. The resulting experimental phase diagram is displayed in Fig. 2(b), scaled in the same way as for the simulations. The inset also displays the tumbling-to-aligning transition lines. As was shown for $f d$-virus suspensions in the fully nematic phase [7], this transition corresponds to the point in the flow curve where shear thickening occurs, as well as
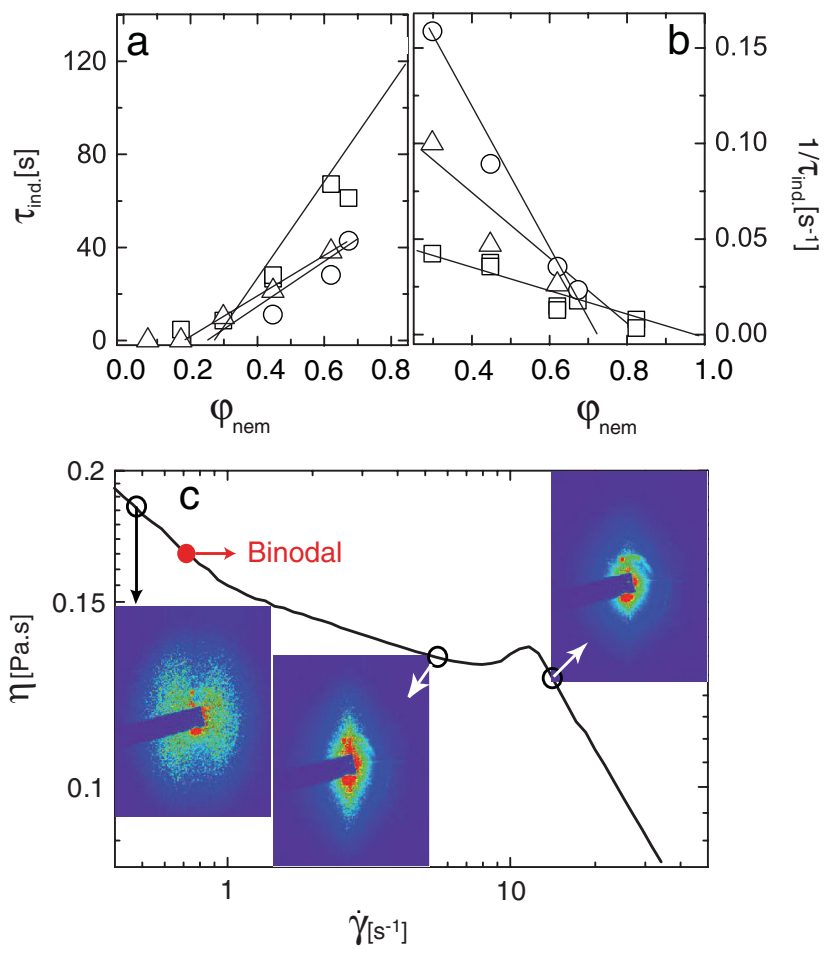

FIG. 3 (color). (a) Induction time and (b) inverse of the induction time after a shear-rate quench from $100 \mathrm{~s}^{-1}$ to $0(\square), 0.5$ $(\bigcirc)$, and $1.5 \mathrm{~s}^{-1}(\triangle)$ as a function of the fraction of nematic phase. Lines correspond to the extrapolated linear behavior. (c) Flow curve of a $f d$-dextran mixture with an equilibrium nematic volume fraction of $62 \%$. The insets are snapshots of light scattering patterns at the indicated shear rates taken after $170 \mathrm{sec}$. All data correspond to a dextran concentration of $18 \mathrm{~g} / 1$.

the shear rate at which nematic domains disappear. Both features can also be observed for flow-induced nematics with $\varphi_{\text {nem }}>0.4$. An example of a flow curve is given in Fig. 3(c). The middle inset in Fig. 3(c) shows an anisotropic scattering pattern typical for the sheared nematic structure, while after the peak in the viscosity only background scattering is observed (right inset). The tumblingto-aligning transition lines obtained from these two different experiments coincide within experimental error. Note that the formation of vorticity bands reported earlier [21] does not affect the light-scattering results.

The most striking feature of Fig. 2 is that the scaled experimental and simulation binodals overlap for different attractions, as do the scaled spinodals. Coexisting nematic states are in tumbling motion while the paranematic state is flow aligned, so that the interface between the two phases is highly dynamic. Time-averaged stresses across such interfaces must be obtained from dynamical equations for order parameters, together with an expression for the stress in terms of these order parameters, in order to calculate an effective interfacial tension. Moreover, the tumbling-toaligning transition line ends at the maximum of the binodal, which is also the point where the tumbling nematic 


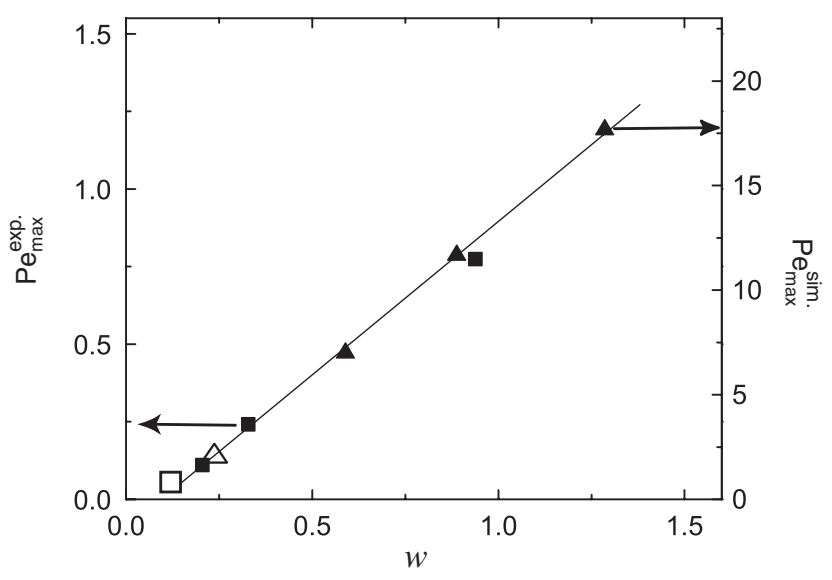

FIG. 4. The Peclet number at the maximum $P_{\max }$ as a function of the width of the equilibrium phase diagram $w$ for simulations ( $\boldsymbol{\Delta}$, right axis) and experiments ( $\boldsymbol{\square}$, left axis). The open symbols denote the extrapolation to the limit of hard rods, with $w=0.1$ for an aspect ratio of 120 ( $\square$, left axis) and $w=0.25$ for an aspect ratio of 20 ( $\triangle$, right axis).

and flow-aligned paranematic states merge. The spinodal line ends far below the maximum of the binodal. The spinodal displays the expected shear-rate dependence; i.e., shear stabilizes the nematic phase, in line with earlier experiments [5]. Note that the experiments show a nonmonotonic concentration dependence of the location of the maximum of the binodal as a function of the strength of attraction contrary to the simulations. This is probably due to the different forms of the interaction potentials in simulations and experiments.

Because of the observed scaling, the effect of attractive interactions on the phase diagram is reduced to two parameters, $\dot{\gamma}_{\max }$ and the biphasic gap width $w$ in the absence of flow, i.e., the difference in packing fractions between the isotropic and nematic phase in equilibrium, $w=\left(\phi_{n}-\right.$ $\left.\phi_{i}\right) / \phi_{0}$ (with $\phi_{0}$ the overall packing fraction). These two parameters are found to be linearly related, as shown in Fig. 4. Here, the bare rotational Peclet numbers are defined as $P e_{\max }=\dot{\gamma}_{\max } / D_{r}^{0}$, where $D_{r}^{0}$ is the rotational diffusion coefficient at infinite dilution. For $f d$ virus, $D_{r}^{0}$ is taken from Ref. [7], while for simulations it is calculated from the length and diameter of a rod [22]. The difference in slopes in Fig. 4 between the experimental and simulation results is due to different aspect ratios of the rods, which affects the rotational self-diffusion close to the phase transition [23]. The open symbols in Fig. 4 refer to an extrapolation to semiflexible rods with only hard-core repulsive interactions.

In conclusion, we have been able to establish generic features of the phase behavior of (attractive) rodlike colloids under flow conditions. Binodals and spinodals in the shear-concentration plane can be mapped onto a master curve for various strengths of attractions. Furthermore, the coexisting nematic is in tumbling motion, which explains why the tumbling-to-aligning transition line in the homo- geneous state at higher shear rates ends at the maximum of the binodal.

We thank K. Mussawisade for his collaboration in the initial stage of this project. Financial support by the German Research Foundation (DFG) within SFB TR 6, and the European Network of Excellence SoftComp (Contract No. NMP3-CT-2004-502235) is gratefully acknowledged. M. R. thanks the Spanish MCYT (Project No. FIS2007-65869-C03-03) for partial support.

*m.ripoll@fz-juelich.de

[1] B. J. Ackerson and P. N. Pusey, Phys. Rev. Lett. 61, 1033 (1988); P. D. Olmsted and P. M. Goldbart, Phys. Rev. A 46, 4966 (1992).

[2] M. C. Cross and P. C. Hohenberg, Rev. Mod. Phys. 65, 851 (1993).

[3] S. Butler and P. Harrowell, Nature (London) 415, 1008 (2002).

[4] S. Hess, Z. Naturforsch. A 31, 1034 (1976); H. See et al., J. Chem. Phys. 92, 792 (1990); P. D. Olmsted and C. Y. D. Lu, Phys. Rev. E 60, 4397 (1999).

[5] T. A. J. Lenstra et al., J. Chem. Phys. 114, 10151 (2001).

[6] R. Larson, Macromolecules 23, 3983 (1990).

[7] M. P. Lettinga et al., Langmuir 21, 8048 (2005).

[8] J.-F. Berret et al., Europhys. Lett. 32, 137 (1995); T. Tiele et al., J. Rheol. (N.Y.) 45, 29 (2001).

[9] E. K. Hobbie and D. J. Fry, Phys. Rev. Lett. 97, 036101 (2006).

[10] J.-F. Berret et al., J. Phys. II (France) 4, 1261 (1994); J.-F. Berret, Langmuir 13, 2227 (1997).

[11] N. Grizzuti and P. L. Maffeteone, J. Chem. Phys. 118, 5195 (2003).

[12] Z. Dogic et al., Phys. Rev. E 69, 051702 (2004).

[13] M.P. Lettinga and J. K. G. Dhont, J. Phys. Condens. Matter 16, S3929 (2004).

[14] G. Germano and F. Schmid, J. Chem. Phys. 123, 214703 (2005).

[15] R. G. Winkler et al., J. Phys. Condens. Matter 16, S3941 (2004).

[16] A. Malevanets and R. Kapral, J. Chem. Phys. 110, 8605 (1999); T. Ihle and D. M. Kroll, Phys. Rev. E 63, 020201 (R) (2001); M. Ripoll et al., Europhys. Lett. 68, 106 (2004).

[17] M. Ripoll et al., Phys. Rev. Lett. 96, 188302 (2006); H. Noguchi and G. Gompper, Proc. Natl. Acad. Sci. U.S.A. 102, 14159 (2005).

[18] A. Yethiraj and H. Fynewever, Mol. Phys. 93, 693 (1998).

[19] See EPAPS Document No. E-PRLTAO-101-038842 for movies of the dynamics $I-N$ system under shear from simulations and experiments. For more information on EPAPS, see http://www.aip.org/pubservs/epaps.html.

[20] Y. G. Tao et al., Phys. Rev. Lett. 95, 237802 (2005).

[21] K. Kang et al., Phys. Rev. E 74, 026307 (2006).

[22] M. M. Tirado and J. García de la Torre, J. Chem. Phys. 73, 1986 (1980).

[23] I. Teraoka and R. Hayakawa, J. Chem. Phys. 91, 2643 (1989). 